\title{
Survey on Energy-Efficient Routing Algorithms for Underwater Wireless Sensor Network
}

\author{
Mukhtiar Ahmed \\ Quaid-e-Awam University, \\ of Science and Technology, \\ Nawabshah, Sindh, Pakistan
}

\author{
Fauzia Talpur, \\ Department of Computer \\ Science, Faculty of Computing \\ UTM, Malaysia
}

\author{
M.Ali Soomro \\ Quaid-e-Awam University, \\ of Science and Technology, \\ Nawabshah, Sindh, Pakistan
}

\begin{abstract}
In underwater environment, for retrieval of information the routing mechanism is used. In routing mechanism there are three to four types of nodes are used, one is sink node which is deployed on the water surface and can collect the information, courier/super/AUV or dolphin powerful nodes are deployed in the middle of the water for forwarding the packets, ordinary nodes are also forwarder nodes which can be deployed from bottom to surface of the water and source nodes are deployed at the seabed which can extract the valuable information from the bottom of the sea. In underwater environment the battery power of the nodes is limited and that power can be enhanced through better selection of the routing algorithm. This paper focuses the energy-efficient routing algorithms for their routing mechanisms to prolong the battery power of the nodes. This paper also focuses the performance analysis of the energy-efficient algorithms under which we can examine the better performance of the route selection mechanism which can prolong the battery power of the node.
\end{abstract}

Keywords: super node; dolphin node; acoustic channel; data packets; forwarder node; ordinary node

\section{INTRODUCTION}

The underwater equipment such as underwater sensor nodes, Acoustic Underwater Vehicles (AUVs) and underwater modems with acoustic channel have made communication possible in the underwater environment. Sensor nodes are able to transmit the data packets with their sensing capabilities within the short distance [1-3]. The underwater sensor nodes are composed of sensing unit, acoustic modem, processing unit, communication unit and power unit. The sensing unit measures the physical conditions like water temperature and water pressure. The acoustic modem is responsible to convert the RF signal into acoustic signaling while processing unit is responsible to process the data and converting it into the required signaling form [4] . The communication unit is used to transfer the data to the acoustic modem. All the discussed units' runs under the power unit, power unit is responsible to supply the required energy to all these units to perform functionality of the node. The nodes in underwater environment have ability to communicate between each other or to communicate with surface nodes through acoustic channel [5]. Majority of the researchers have given feasible algorithms, deployment methodologies, different architectural structures as well as data forwarding mechanisms to prolong the battery life of the nodes; but due to the underwater environmental conditions and some delay factors, the underwater nodes cannot maintain their power levels and the power supply deplete earlier [6]. Following are the energyaware routing protocols described with advantages and drawbacks.

\section{RELATED WORK}

Delay-tolerant Data Dolphin (DDD) is an energy efficient routing algorithm proposed by Magistretti, et al. [7]. DDD is for the delay tolerant applications. The DDD routing algorithm is based on collector nodes called dolphin and stationary nodes; the dolphin nodes harvest the information sensed by the stationary nodes. The routing algorithm eliminates the energy expensive multi-hop communication. The stationary nodes are responsible to transmit its collected data to the nearest in the range of dolphins. The stationary nodes are deployed on sea bed area of interest. The authors have used the two components of the acoustic channel one is communication component and other is transceivers component for data forwarding. Through communication component the dolphin node is able to communicate and through transceivers component the presence of dolphin node is analyzed through beacon signal. The dolphin node forwards the collected packets to the base station which are deployed on water surface. In DDD the random movement of dolphin nodes will not able to collect all the data packets from the sensor nodes and in resultant the data delivery ratio will be degraded. In DDD; if the number of dolphins increases the overall cost of the network will also be increased.

Power-Efficient Routing (PER) proposed by Huang, et al. [8]. PER routing enhances the battery power of the sensor node. PER algorithm is based on two modules one is forwarder node selector and other is forwarding tree trimming. In PER the fuzzy logic system and decision trees based mechanisms are used for forwarder node selector, the forwarder node selection mechanism may be affected due to the water pressure and may reduce the data delivery ratio of PER. If forwarder node will come in the void region then it will drop the packets continuously and will die earlier.

Energy Efficient Depth Based Routing (EEDBR) is proposed by Wahid et al. [9]. EEDBR protocol is based on knowledge acquisition and data forwarding phases. In EEDBR ordinary sensor nodes are placed from top to bottom of the water and sink nodes are placed on water surface and source nodes are placed at bottom of the water. In EEDBR from sink nodes to onshore data center the radio signaling are used whereas in underwater the acoustic signaling is used. In knowledge acquisition phase the Hello message is forwarded between sensor nodes and neighbor nodes the nodes which keep smaller depth Id may involve for data forwarding. In data forwarding mechanism the depth and residual energy parameters are considered. The nodes which keep high energy with smaller depth are only involved in data forwarding. The depth calculation mechanism adapted by authors is failure in 
sparse area network. No any proper algorithm is given by EEDBR for balanced energy consumption.

Energy efficient Mobicast routing protocol is proposed by Chen and Lin [10]. The Mobicast is power-saving 3D routing protocol which overcomes the problem of unpredictable 3D holes. In Mobicast the "apple peel" is proposed to resolve the problem of unpredictable 3D holes. The architecture of Mobicast is based on underwater sensor nodes which are deployed randomly in 3D area of water around the Autonomous Underwater Vehicles (AUV) in form of 3D zone of reference or 3D ZOR as shown in Figure 1 and Figure 2. The AUV travels along the user defined path and collects the information from the sensor nodes within different time intervals of 3D ZORs. The sleep and active nodes are used to resolve the problem of unpredictable holes. Active nodes are responsible to forward the sensed data to the AUV. Mobicast uses the geographic 3D Zone of Relevance (3D ZOR3) and 3D Zone of Forwarding (3D ZOF) which are created by AUV at time $\mathrm{t}$ to indicate which sensor node should forward the sensed data to the AUV as shown in Figure 1 and Figure 2. Fully distributed algorithm is used by Mobicast which reduces the power consumption of the sensor nodes and the message overheads. Mobicast enhances the data delivery ratio. In Mobicast, the creation of user defined route in underwater 3D environment is very hard due to the water pressure. Due to the continuous water movement if the active nodes may remain away from the AUV then the active nodes can drop the packets and ultimately the packets delivery ratio will be reduced.

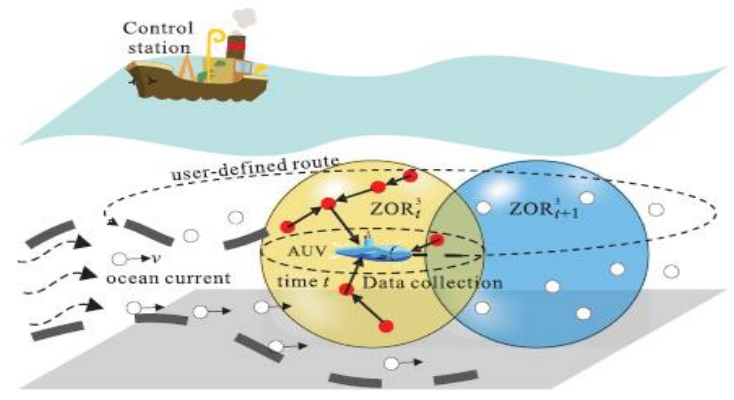

Figure 1: AUV collects data from sensor nodes with [ZOR】_t^3 with time $\mathrm{t}[10]$

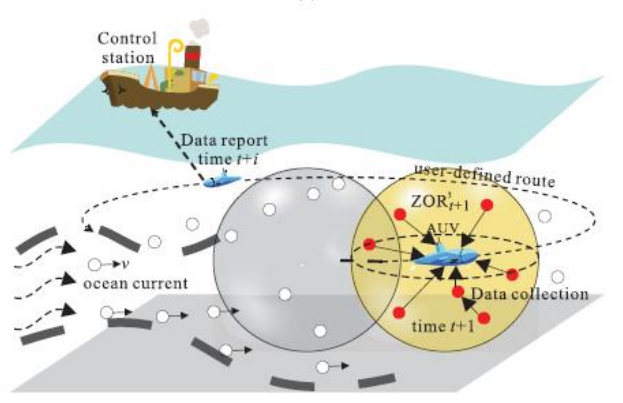

Figure 2: AUV collects data from sensor nodes with [ZOR】_t^3 with time $\mathrm{t}+1[10]$

Link-state Adaptive Feedback Routing (LAFR) algorithm is proposed by Zhang, et al. [11]. LAFR is energy efficient routing algorithm based on asymmetric link mechanism. The data forwarding mechanism is based on beam width with communication range up to 3600 angle. The complicated mechanism for data forwarding with calculation of angle will put the heavy load on ordinary sensor node; due to this network load the sensor node will die earlier and average energy consumption of LAFR will be increases.

Multi-layer Routing Protocol (MRP) is proposed by Wahid, et al. [12]. MRP routing protocol is used to resolve the problem of localization and enhances the battery life of ordinary sensor node. The network architecture is based on sink nodes, super nodes and sensor nodes. The sink nodes are placed on the water surface and super nodes are fixed and spread in different water levels. Sensor nodes are deployed at the bottom of the water. MRP develops the 2D layers around the super node. For packets forwarding the layer ID and sensor node ID with different power levels are proposed, the creation of layers are shown in Figure 3. The authors claimed that the super node is used to enhance the battery life of ordinary sensor nodes. MRP uses the 2D node deployment mechanism but underwater supports 3D deployment. In MRP the packets holding time algorithm is not properly defined. If sensor node may remain away from super node then the sensor node may drop the packets and ultimately the packets delivery ratio might be affected.

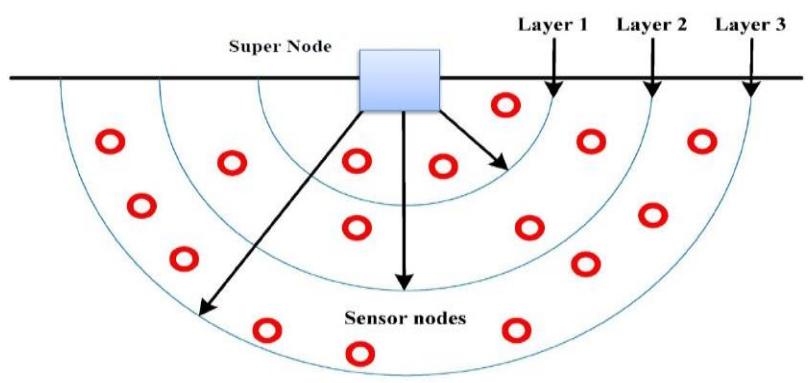

Figure 3: Formation of different layers around super node [12]

Energy Efficient Depth Based Routing (EE-DBR) is proposed by Diao, et al. [13] which prolongs the battery life of the sensor node through reducing of multipath redundancy forwarding mechanism. The ToA ranging technique is used to measure distance between sensor nodes. If measured distance increases the node will stop the packets forwarding and can save its energy. ToA with measured distance technique of EEDBR controls the data packets on a fixed range data forwarding route, if distance increases from source to sink node the ToA with measured distance technique will enhance the end-to-end delay.

Reliable and Energy Efficient Protocol (REEP) is proposed by Rahman, et al. [14] which utilizes the Time of Arrival (ToA) algorithms as described by Diao, et al. [13] to find the node distance to its respective sink. REEP utilizes the ToA information to find best available routing path. A REEP operation is based on two phases one is network setup phase and other is data transmission phase. In network setup phase the sink nodes are deployed on water surface whereas ordinary nodes are deployed in underwater. In network phase forwarder node selection is based on node location information and node residual energy. In REEP the ToA arrival technique is used to find the approximately distance between sender and receiver nodes. The three kind of packets format are used to develop the route for packets forwarding: 
one is hello packet format, second is reply packet format, and third is data packet format. In data transmission phase data packets are forwarded through multi-hop with calculated distance from source to destination. The REEP packets forwarding functionality is based only on vertical modem; if majority number of nodes become sparse the distance calculation mechanism of REEP will become failure and nodes will drop the packets continuously and will lose their energy.

Energy-efficient Multipath Grid-based Geographic Routing (EMGGR) is proposed by Al Salti, et al. [15]. EMGGR is position based routing algorithm and is consists of 3D logical grid multipath approach. The EMGGR refers the three phases one is gateway election mechanism, second is updating gateways' information mechanism, and third is packets forwarding mechanism. EMGGR considers the geographic area of the network with 3D logical grid partitions. EMGGR considers the deployment of every sensor node with the single cell from the logical grid with xyz addressing mechanism and presumes that every sensor node is well aware about its location through built-in localization service. EMGGR is based on multipath route selection from source to virtual cell gateway node and virtual cells gateway nodes are responsible to relay the data packets to the surface sink node. The algorithm is based on single sink node only.

Energy-efficient Distance Routing Protocol (DRP) proposed by Chao, et al. [16] considers the distance-varied collision probability for route selection with residual energy of each node. DRP is based on smaller and larger vulnerability range between nodes through inner and outer radius. In DRP the sink node broadcast HELLO packets periodically to the neighbor nodes and the best forwarder node can be selected through its residual energy. In DRP the multipath node disjoint route is developed between sink and source nodes through forwarder nodes. In DRP if the number of nodes increases the performance of entire network is reduces due collision probability. In DRP if the distance will increase due to mobile node then forwarder node will drop the packets and will die earlier.

\section{PERFORMANCE ANALYSIS}

In Table 1, the parametric performance analysis of the energy efficient routing algorithms is shown, the performance parameters are selected from the architecture and operation of the energy efficient routing algorithms. The parameters for measuring the performance of the energy efficient routing algorithms are: single/multiple copies, hop-by-hop/end-toend, single/multiple sink, multipath, hello/control packet, and requirements/assumptions

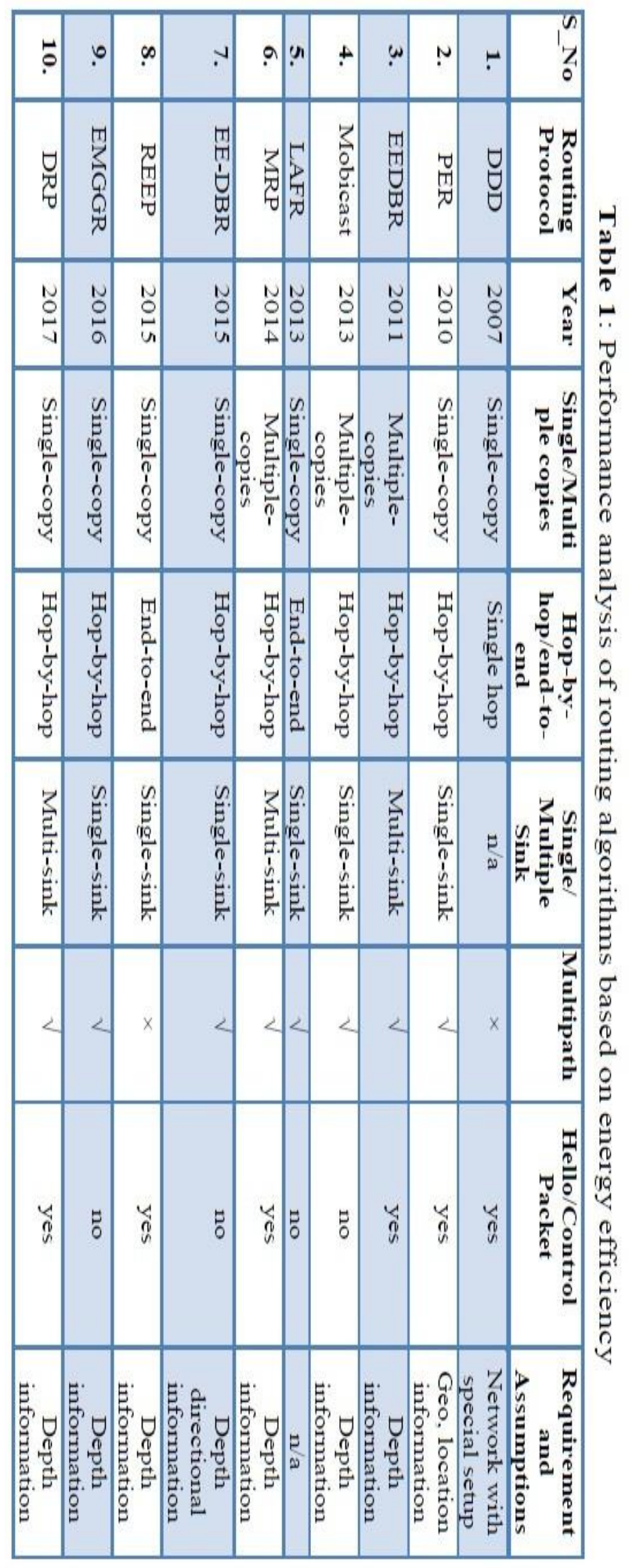

\section{CONCLUSION}

Energy-efficient routing algorithms as mentioned above are based to prolong the battery power of the sensor nodes, DDD algorithm has used the dolphin node to save the power of the nodes, PER algorithm has used the fuzzy logic and decision tree approach to prolong the battery power of the node, EEDBR saves the energy of the nodes through forwarder node selection and residual energy selection mechanism, Mobicast 
prolong the battery power of the node through AUV. LAFR routing algorithm prolong the battery power of the node through asymmetric link selection mechanism, MRP prolongs the battery power of the node through layer-formation and super node selection mechanism. EE-DBR is based on distance selection and ToA arrival which saves the power of the ordinary node. From aforementioned algorithms the EMGGR algorithm uses the 3D grid approach with multipath selection mechanism which is the better approach to prolong the battery power of the nodes as compare to other defined algorithms.

\section{REFERENCES}

[1] M. Ahmed and M. Salleh, "Localization schemes in Underwater Sensor Network (UWSN): A Survey," Indonesian Journal of Electrical Engineering and Computer Science., vol. 1, pp. 119-125, 2015.

[2] M. Z. Abbas, K. A. Bakar, M. A. Arshad, M. Tayyab, and M. H. Mohamed, "Scalable Nodes Deployment Algorithm for the Monitoring of Underwater Pipeline," TELKOMNIKA (Telecommunication Computing Electronics and Control), vol. 14, pp. 1183-1191, 2016.

[3] N. Bahrami, N. H. H. Khamis, A. Baharom, and A. Yahya, "Underwater Channel Characterization to Design Wireless Sensor Network by Bellhop," TELKOMNIKA (Telecommunication Computing Electronics and Control), vol. 14, pp. 110-118, 2016.

[4] M. Ahmed, M. Salleh, and M. I. Channa, "Critical Analysis of Data Forwarding Routing Protocols Based on Single path for UWSN," International Journal of Electrical and Computer Engineering, vol. 6, pp. 16951701,2016

[5] G. Han, J. Jiang, N. Bao, L. Wan, and M. Guizani, "Routing protocols for underwater wireless sensor networks," Communications Magazine, IEEE, vol. 53, pp. 72-78, 2015.

[6] N. Li, J.-F. Martínez, J. M. Meneses Chaus, and M. Eckert, "A Survey on Underwater Acoustic Sensor Network Routing Protocols," Sensors, vol. 16, p. 414, 2016.

[7] E. Magistretti, J. Kong, U. Lee, M. Gerla, P. Bellavista, and A. Corradi, "A mobile delay-tolerant approach to long-term energy-efficient underwater sensor networking," in Wireless Communications and Networking Conference, 2007. WCNC 2007. IEEE, Sheraton Hotel, Hong Kong, 2007, pp. 2866-2871.

[8] C. J. Huang, Y. W. Wang, H. H. Liao, C. F. Lin, K. W. $\mathrm{Hu}$, and T. Y. Chang, "A power-efficient routing protocol for underwater wireless sensor networks," Applied Soft Computing, vol. 11, pp. 2348-2355, Mar 2011.

[9] A. Wahid, S. Lee, H. J. Jeong, and D. Kim, "EEDBR: Energy-Efficient Depth-Based Routing Protocol for Underwater Wireless Sensor Networks," Advanced Computer Science and Information Technology, vol. 195, pp. 223-234, 2011.

[10] Y.-S. Chen and Y.-W. Lin, "Mobicast routing protocol for underwater sensor networks," Sensors Journal, IEEE, vol. 13, pp. 737-749, 2013.

[11] S. Zhang, D. Li, and J. Chen, "A link-state based adaptive feedback routing for underwater acoustic sensor networks," Sensors Journal, IEEE, vol. 13, pp. 44024412, 2013.

[12] A. Wahid, S. Lee, D. Kim, and K. S. Lim, "MRP: A Localization-Free Multi-Layered Routing Protocol for Underwater Wireless Sensor Networks," Wireless Personal Communications, vol. 77, pp. 2997-3012, Aug 2014.

[13] B. Diao, Y. Xu, Z. An, F. Wang, and C. Li, "Improving Both Energy and Time Efficiency of Depth-Based Routing for Underwater Sensor Networks," International Journal of Distributed Sensor Networks, vol. 11, pp. 1-9, 2015.

[14] Z. Rahman, F. Hashim, M. Othman, and M. F. A. Rasid, "Reliable and energy efficient routing protocol (REEP) for underwater wireless sensor networks (UWSNs)," in Communications (MICC), 2015 IEEE 12th Malaysia International Conference on, Kuching, Sarawak, Malaysia, 2015, pp. 24-29.

[15] F. Al Salti, N. Alzeidi, and B. R. Arafeh, "EMGGR: an energy-efficient multipath grid-based geographic routing protocol for underwater wireless sensor networks," Wireless Networks, vol. 23, pp. 1301-1314, 2017.

[16] C. M. Chao, C. H. Jiang, and W. C. Li, "DRP: An energy-efficient routing protocol for underwater sensor networks," International Journal of Communication Systems, vol. 1, pp. 1-24, 2017. 